\title{
Sampling on the simplex
}

\author{
P.G.L. Porta Mana $\odot$ \\ Kavli Institute, Trondheim <pgleportamana.org> \\ 14 July 2019; updated 4 June 2020
}

\begin{abstract}
Two simple approaches to sampling general probability distributions defined over the simplex, especially with Monte Carlo algorithms and in high dimensions, are presented. The approaches can be generalized to spaces bounded by linear constraints.
\end{abstract}

Dedicated to Nerone. We still miss you every day.

The need to sample from a general distribution of probability over a simplex, that is, over a space of distributions, is more common than one might think. For example, it's necessary whenever we use exchangeable probability models ${ }^{1}$, ubiquitous in science owing to their intimate connection with reproducibility ${ }^{2}$.

Sampling on an $n$-dimensional simplex, for example by Monte Carlo methods ${ }^{3}$, is difficult because the allowed ranges of the sampled quantities are interdependent: denoting by $f:=\left(f_{0}, \ldots, f_{n}\right)$ a generic sample, we must have

$$
\sum_{i=0}^{n} f_{i}=1, \quad f_{i} \geqslant 0, \quad \text { all } i \in\{0, \ldots, n\},
$$

so that $n$ independent components, for example $\boldsymbol{F}:=\left(f_{1}, \ldots, f_{n}\right)$, must satisfy

$$
\sum_{l=1}^{n} f_{l} \leqslant 1, \quad f_{l} \geqslant 0, \quad \text { all } l \in\{1, \ldots, n\} .
$$

In the following, $i$ always runs from 0 to $n$, and $l$ from 1 to $n$.

Individual bounds $\left\{0 \leqslant f_{l} \leqslant 1\right\}$ are not difficult to handle with a change of variable, using a logistic function for example, because they are independent. It's the joint bound from the sum in (2) that's the problem. It needs to be tested for any proposed sample, and in high

1 Dawid 2013; de Finetti 1930; 1937; Bernardo \& Smith 2000 §4.2. $\quad 2$ JCGM 2008 $\S \S$ B.2.15-16; ISO $2006 \S \S 3.3 .6,3.3 .11$; Poincaré 1992 ch. IX; Duhem 1914 ch. VI; de Finetti 1937; Jeffreys 1973 ch. IV; Popper 2005 ch. $1 . \quad{ }^{3}$ Neal 1993; MacKay 2003 chs 29, 30; Hall 2014. 
dimensions it leads to high rejection rates, because the $n$-dimensional simplex, considered as embedded in the positive orthant of $\mathbf{R}^{n}$, only occupies $1 / n$ ! of the volume of the unit hypercube $\left\{0 \leqslant f_{l} \leqslant 1\right\}$.

I didn't find solutions to this problem in the literature, except for an ingenious change of variables $z \mapsto \boldsymbol{F}$, discussed by Ferguson et al. ${ }^{4}$ and Betancourt (2012; Altmann et al. 2014), which maps the unit hypercube onto (but not into) the simplex:

$$
\begin{aligned}
f_{1} & =1-z_{1} \\
f_{2} & =\left(1-z_{2}\right) z_{1} \\
f_{3} & =\left(1-z_{3}\right) z_{2} z_{1} \\
& \cdots \\
f_{n} & =\left(1-z_{n}\right) z_{n-1} \cdots z_{1} \\
{\left[f_{0}\right.} & \left.\equiv z_{n} \cdots z_{1}\right] .
\end{aligned}
$$

You can easily check that the quantities $z:=\left(z_{1}, \ldots, z_{n}\right)$ have independent bounds $\left\{0 \leqslant z_{l} \leqslant 1\right\}$. The Jacobian determinant of this transformation can also be computed. This transformation has a couple of practical disadvantages, however, owing to its recursive structure. The numerical relative imprecision of the $z_{l}$ accumulates in the $f_{l}$ as $l$ increases, leading to an additional variability in the $f_{l}$ with larger $l$, which manifests as an artefactual broadening of their marginal densities. The non-linear transformation $z \mapsto \boldsymbol{F}$ moreover destroys the convex structure of the simplex; it heavily bends regions that are linear in the $f_{l}$, and can thus lead to a slower exploration of bent regions of high probability for some Markov-chain methods.

A more general version of this problem appears for spaces bounded by linear or affine inequalities of the type

$$
\left\{\sum_{l=1}^{n} A_{c l} f_{l} \leqslant b_{c}\right\}
$$

I want to propose two alternative approaches to the problem of interdependent bounds (2). These approaches work very well in high dimensions. They are so simple - the first in particular - that they have probably been used before, so I'm not making any claim of originality. But I haven't seen them explicitly discussed in the literature, so it may

\footnotetext{
${ }^{4}$ Ferguson et al. 1992.
} 
be useful to advertise them. The main idea behind them is to give an additional dimension to the simplex; more precisely, to sample in a space of which the simplex is a central projection. These approaches can be extended to more general bounds like (4).

Suppose we want to sample from the probability distribution

$$
\begin{gathered}
G(\boldsymbol{F}) \mathrm{d} \boldsymbol{F} \\
f_{l} \geqslant 0, \quad \sum_{l=1}^{n} f_{l} \leqslant 1, \quad\left[f_{0} \equiv 1-\sum_{l=1}^{n} f_{l}\right],
\end{gathered}
$$

defined over the $n$-dimensional simplex. This distribution can be considered as the marginal distribution of the joint, separable distribution

$$
L(r) G(\boldsymbol{F}) \mathrm{d} r \mathrm{~d} \boldsymbol{F}, \quad r \geqslant 0, \quad f_{l} \geqslant 0, \quad \sum_{l=1}^{n} f_{l} \leqslant 1,
$$

where $r$ is a positive quantity with a (regular) distribution of probability $L(r) \mathrm{d} r$. If we sample $(r, \boldsymbol{F})$ from this joint distribution, the samples of $\boldsymbol{F}$ will obviously come from $G(\boldsymbol{F}) \mathrm{d} \boldsymbol{F}$.

First approach Now consider the quantities $x:=\left(x_{0}, \ldots, x_{n}\right)$ in the non-negative $(n+1)$-dimensional orthant $\left\{x_{i} \geqslant 0\right\}$, origin excluded, $\boldsymbol{x} \neq \mathbf{0}$, and the following transformation $\boldsymbol{x} \mapsto(r, \boldsymbol{F})$ :

$$
\begin{gathered}
r=x_{0}+\cdots+x_{n} \\
f_{l}=\frac{x_{l}}{x_{0}+\cdots+x_{n}} \\
{\left[f_{0} \equiv \frac{x_{0}}{x_{0}+\cdots+x_{n}}\right] .}
\end{gathered}
$$

You can prove, for example using the inverse transformation $(r, \boldsymbol{F}) \mapsto \boldsymbol{x}$, that the Jacobian determinant of $x \mapsto(r, F)$ is $r^{-n}$. The joint probability distribution (6) can thus be written as

$$
M[r(x)] G[F(x)] \mathrm{d} x, \quad x_{i} \geqslant 0,
$$

where the Jacobian determinant has been absorbed into $M(r):=L(r) / r^{n}$. 
Most important, the $x_{i}$ have independent bounds: if $x_{i} \geqslant 0$ and $x \neq 0$, the interdependent bounds (2) are automatically satisfied, and vice versa:

$$
\begin{gathered}
f_{l} \equiv \frac{x_{l}}{x_{0}+\cdots+x_{n}} \geqslant 0 \quad \Longleftrightarrow \quad x_{l} \geqslant 0 \\
\sum_{l=1}^{n} f_{l} \equiv \frac{\sum_{l=1}^{n} x_{l}}{x_{0}+\sum_{l=1}^{n} x_{l}} \leqslant 1 \quad \Longleftrightarrow \quad x_{0} \geqslant 0 .
\end{gathered}
$$

We can therefore easily sample from the distribution (8) for the quantities $x$, on a space with no interdependent bounds, and then construct samples of $\boldsymbol{F}$ (and therefore $f$ ) from $x$ via the transformation (7b). The resulting $\boldsymbol{F}$ samples are drawn from our distribution of interest (5). The $r$ samples are drawn from $L(r) \mathrm{d} r$ but they aren't calculated (unless this quantity is of interest for some reason). Note that $L$ and therefore $M$ are completely arbitrary; we can choose a normal distribution as the latter, for example. Also, the numerical relative imprecision of every $f_{i}$ is roughly twice that of $x_{i}$ : no accumulation of imprecision occurs. We have increased the dimension of our sample space by one, but the cost of sampling the extra dimension can be more than repaid by the gain of avoiding interdependent bounds like (2).

If our probability distribution (5) has its main mass enough far away from the faces of the simplex, it's practical to sample the $x$ directly despite their lower bound - by using a density function $M(r)$ with a sharp peak at high values of $r$, say $r \approx n$. This way, even if some typical $f_{i}$ may be close to zero, the corresponding $x_{i}$ will be of order unity, enough far away from its lower bound to be sampled directly without worrying about high rejection rates.

This approach can be generalized to bounds like (4) by introducing an extra dimension per constraint and rescaling.

Second approach If we prefer to have completely unbounded quantities, we can still consider the probability distribution (6) extended with $r$, but 
use the following transformation $\boldsymbol{t} \mapsto(r, \boldsymbol{F})$ with $t \in \mathbf{R}^{n+15}$ :

$$
\begin{gathered}
r=t_{0}+\cdots+t_{n} \\
f_{l}=\frac{\exp \left(t_{l}\right)}{\exp \left(t_{0}\right)+\cdots+\exp \left(t_{n}\right)} \\
{\left[f_{0}=\frac{\exp \left(t_{0}\right)}{\exp \left(t_{0}\right)+\cdots+\exp \left(t_{n}\right)}\right] .}
\end{gathered}
$$

You can prove, for example by induction on $n$, that the Jacobian determinant of the transformation above is

$$
\left|\operatorname{det} \frac{\partial(r, \boldsymbol{F})}{\partial \boldsymbol{t}}\right|=(n+1) \prod_{i=0}^{n} f_{i} \equiv(n+1) \exp (r)\left[\sum_{i} \exp \left(t_{i}\right)\right]^{-(n+1)} \text {. }
$$

The joint probability distribution (6) can thus be written as

$$
(n+1) L[r(\boldsymbol{t})] G[\boldsymbol{F}(\boldsymbol{t})] \prod_{i=0}^{n} f_{i}(\boldsymbol{t}) \mathrm{d} \boldsymbol{t}, \quad \boldsymbol{t} \in \mathbf{R}^{n+1} .
$$

We can therefore sample from the distribution (12), defined over $\mathbf{R}^{n+1}$, and then construct samples for $\boldsymbol{F}$ and $\boldsymbol{f}$ from $\boldsymbol{t}$ via the transformation (10b). Also in this case the resulting $\boldsymbol{F}$ samples are drawn from our distribution of interest (5). The probability density function $L(r)$ is again completely arbitrary, and can be conveniently chosen as a normal sharply peaked at $r \approx 0$; it's also possible to include the Jacobian term $\exp (r)$ from (11) in it. This approach can also be extended to general bounds like (4).

A final remark: instead of the transformation (10a) we could also have considered $r=\sum_{i} \exp \left(\boldsymbol{t}_{i}\right)$. This transformation is inconvenient, however: surfaces of constant $r$ would then be heavily bent in $t$ coordinates, leading to slower convergence for some Markov-chain methods. It's also possible to use the transformation (10b) without $r$, by using the independent quantities $\left(t_{1}, \ldots, t_{n}\right)$ and defining $t_{0}:=-\sum_{l=1}^{n} t_{l}$. The Jacobian determinant in this case is still (11); the proof is left again as an exercise for you.

\section{Thanks}

... To Iván Davidovich for a useful discussion. To Mari \& Miri for continuous encouragement and affection. To Buster Keaton and Saitama

5 cf. MacKay 2003 § 23.5, p. 316. 
for filling life with awe and inspiration. To the developers and maintainers of $\mathrm{AT}_{\mathrm{E}} \mathrm{X}$, Emacs, AUCTEX, Open Science Framework, Python, Inkscape, Sci-Hub for making a free and impartial scientific exchange possible. $\mathbf{x}$ This work is financially supported by the Kavli Foundation and the Centre of Excellence scheme of the Research Council of Norway.

\section{Bibliography}

('de $X^{\prime}$ is listed under $\mathrm{D}$, 'van $X^{\prime}$ under $\mathrm{V}$, and so on, regardless of national conventions.)

Altmann, Y., McLaughlin, S., Dobigeon, N. (2014): Sampling from a multivariate Gaussian distribution truncated on a simplex: a review. IEEE Workshop Stat. Signal Process. 2014, 113-116. http://dobigeon.perso.enseeiht.fr/papers/Altmann_IEEE_SSP_2014.pd $f$. Note: the 'covariance' matrices used in $\S 5$ of this paper are not positive-definite.

Bernardo, J.-M., Smith, A. F. (2000): Bayesian Theory, repr. (Wiley, New York). First publ. 1994.

Betancourt, M. (2012): Cruising the simplex: Hamiltonian Monte Carlo and the Dirichlet distribution. Am. Inst. Phys. Conf. Proc. 1443, 157-164.

Damien, P., Dellaportas, P., Polson, N. G., Stephens, D. A., eds. (2013): Bayesian Theory and Applications. (Oxford University Press, Oxford).

Dawid, A. P. (2013): Exchangeability and its ramifications. In: Damien, Dellaportas, Polson, Stephens (2013), ch. 2, 19-29.

de Finetti, B. (1930): Funzione caratteristica di un fenomeno aleatorio. Atti Accad. Lincei: Sc. Fis. Mat. Nat. IV 5 , 86-133. http://www. brunodefinetti.it/0pere.htm.

- (1937): La prévision : ses lois logiques, ses sources subjectives. Ann. Inst. Henri Poincaré $7^{1}$, 1-68. Transl. in Kyburg, Smokler (1980), pp. 53-118, by Henry E. Kyburg, Jr.

Duhem, P. (1914): La Théorie Physique : son objet - sa structure, 2nd ed. (Marcel Rivière, Éditeur, Paris). http://virtualbooks.terra.com.br/freebook/fran/la_theorie_ph ysique. htm. First publ. 1906. Transl. as Duhem (1991).

- (1991): The Aim and Structure of Physical Theory, transl. of the 2nd ed. (Princeton University Press, Princeton). Transl. of Duhem (1914) by P. P. Wiener. First publ. in French 1906.

Ferguson, T. S., Phadia, E. G., Tiwari, R. C. (1992): Bayesian nonparametric inference. IMS Lecture Notes Monogr. Ser. 17, 127-150.

Hall, B. (2014): MCMC algorithms. https://m-clark.github.io/docs/ld_mcmc/. See also https://github.com/LaplacesDemonR/LaplacesDemon, https://web.archive.org/w eb/20141224051720/http: //Www . bayesian - inference. com/index.

ISO (International Organization for Standardization) (2006): ISO 3534-2:2006: Statistics - Vocabulary and symbols - Part 2: Applied statistics. International Organization for Standardization.

JCGM (Joint Committee for Guides in Metrology) (2008): JCGM 100:2008: Evaluation of measurement data - Guide to the Expression of Uncertainty in Measurement, corr. version. Joint Committee for Guides in Metrology (JCGM), BIPM, IEC, IFCC, ILAC, ISO, IUPAC, IUPAP, OIML. http://www. bipm . org/en/publications/guides/gum. html. Includes various supplements. First publ. 1993.

Jeffreys, H. (1973): Scientific Inference, 3rd ed. (Cambridge University Press, Cambridge). First publ. 1931. 
Kyburg Jr., H. E., Smokler, H. E., eds. (1980): Studies in Subjective Probability, 2nd ed. (Robert E. Krieger, Huntington, USA). First publ. 1964.

MacKay, D. J. C. (2003): Information Theory, Inference, and Learning Algorithms. (Cambridge University Press, Cambridge). http://www. inference. phy.cam.ac.uk/mackay/itila /. First publ. 1995.

Neal, R. M. (1993): Probabilistic inference using Markov chain Monte Carlo methods. Tech. rep. CRG-TR-93-1. (University of Toronto, Toronto). http://www. cs . utoronto. ca/ radfo rd/review. abstract. html, http://omega . albany . edu: 8008/neal . pdf.

Poincaré, H. (1905): Science and Hypothesis. (Walter Scott, London). Transl. of Poincaré (1992) by W. J. Greenstreet; with a Preface by J. Larmor. First publ. 1902. Partly repr. in Poincaré (1958).

- (1958): The Value of Science. (Dover, New York). Authorized transl. with an introduction by G. B. Halsted. First publ. 1913.

- (1992): La science et l'hypothèse. (Éditions de la Bohème, Rueil-Malmaison, France). htt p: //gallica. bnf.fr/document?0=N026745. First publ. 1902; transl. as Poincaré (1905).

Popper, K. (2005): The Logic of Scientific Discovery, repr. (Routledge, London). First publ. in German 1935. 